\title{
How accurate is Limber's equation? (Research Note)
}

\author{
P. Simon ${ }^{1,2}$ \\ ${ }^{1}$ Institute for Astronomy, University of Edinburgh, Royal Observatory, Blackford Hill, Edinburgh EH9-3HJ, UK \\ e-mail: psimon@roe.ac.uk \\ 2 Argelander-Institut für Astronomie ${ }^{\star}$, Universität Bonn, Auf dem Hügel 71, 53121 Bonn, Germany
}

Received 6 September 2006 / Accepted 26 July 2007

\section{ABSTRACT}

\begin{abstract}
Aims. The so-called Limber equation is widely used in the literature to relate the projected angular clustering of galaxies to the spatial clustering of galaxies in an approximate way. This note gives estimates of where the regime of applicability of Limber's equation stops.

Methods. This paper revisits Limber's equation and compares its predictions to the accurate integral of which the Limber equation is an approximation for some realistic situations. Thereby, the accuracy of the approximation is quantified. Different cases of spatial correlation functions are considered.

Results. Limber's equation is accurate for small galaxy separations but breaks down beyond a certain separation that depends mainly on the ratio $\sigma / r_{\mathrm{m}}$ and to some degree on the power-law index of spatial clustering; $\sigma$ is the $1 \sigma$-width of the galaxy distribution in comoving distance, and $r_{\mathrm{m}}$ the mean comoving distance. Limber's equation introduces a $10 \%$ systematic error that may be important at a scale of about a few degrees or in some cases, narrow galaxy distributions for example, even at arcmin scales. An alternative integral for such cases is given inside this paper.
\end{abstract}

Key words. galaxies: fundamental parameters - cosmology: observations - large-scale structure of Universe - cosmology: theory

\section{Introduction}

To relate the projected correlation function of galaxies on the sky to the spatial, three-dimensional correlation functions many authors use an approximation that was introduced by Limber (1953). This approximation is nowadays referred to as Limber's equation.

However, as will be shown in Sect. 2.2, Limber's approximation diverges when galaxies are closely distributed. Therefore, Limber's approximation is bound to break down at some point. Peebles (1980, p. 199) argued that this break-down occurs at separations of roughly $30^{\circ}$, but made assumptions about galaxy surveys that do not meet the standards of many contemporary wide-field surveys. Due to the availability of spectroscopic or photometric redshifts quite narrowly binned distributions are often used in current studies.

This paper delineates the circumstances in which Limber's approximation is valid and gives an alternative for situations where it is not valid. Throughout this paper a flat universe will be assumed.

\section{Relation between spatial and angular correlation function}

\subsection{Exact relation with only some restrictions}

The angular correlation of galaxy clustering, $\omega(\theta)$, which is a function of galaxy separation $\theta$, can be related to the spatial

* Founded by merging of the Institut für Astrophysik und Extraterrestrische Forschung, the Sternwarte, and the Radioastronomisches Institut der Universität Bonn. clustering, $\xi(r, t(r))$ (a function of comoving distance $r$ and cosmic time $t(r)$ ), by cross-correlating the projected galaxy (number) density fields $n$ (e.g. Peebles 1980):

$\omega(\theta) \approx \int_{0}^{\infty} \mathrm{d} r_{1} \int_{0}^{\infty} \mathrm{d} r_{2} p_{1}\left(r_{1}\right) p_{2}\left(r_{2}\right) \xi\left(R, \frac{r_{1}+r_{2}}{2}\right)$,

where

$R \equiv \sqrt{r_{1}^{2}+r_{2}^{2}-2 r_{1} r_{2} \cos \theta}$

The filters $p_{1 / 2}(r)$ project the three-dimensional density contrast, $\delta \equiv \frac{n}{\langle n\rangle}-1$, to the density contrast on the sky:

$\hat{\delta}_{1,2}(\boldsymbol{\theta})=\int_{0}^{\infty} \mathrm{d} r p_{1,2}(r) \delta_{1,2}(r \boldsymbol{\theta}, r)$,

where $r$ is the comoving radial distance along the line-of-sight, $\boldsymbol{\theta}$. Note that Eq. (1) is valid even for large $\theta$.

\subsection{Limber's approximation and its breakdown}

Limber (1953) introduced several approximations in order to simplify the integral Eq. (1). Here only the result is quoted:

$$
\begin{aligned}
\omega(\theta) & =\int_{0}^{\infty} \mathrm{d} \bar{r} p_{1}(\bar{r}) p_{2}(\bar{r}) \int_{-\infty}^{+\infty} \mathrm{d} \Delta r \xi(\hat{R}, \bar{r}) \\
\hat{R} & \equiv \sqrt{\bar{r}^{2} \theta^{2}+\Delta r^{2}} .
\end{aligned}
$$

For convenience the following new coordinates are used:

$\bar{r} \equiv \frac{r_{1}+r_{2}}{2} ; \Delta r \equiv r_{2}-r_{1}$. 
For historical reasons, as a further approximation it is assumed in the above equations that we are dealing with small angles of separation, $\theta$, by which we can introduce: ${ }^{1}$

$1+\cos \theta \approx 2 ; 1-\cos \theta \approx \frac{\theta^{2}}{2} ; R \approx \sqrt{\bar{r}^{2} \theta^{2}+\Delta r^{2}}$.

Usually, when employing Limber's equation this approximation is automatically used.

The useful Eq. (4) is frequently used in the astronomy because it allows one to find an analytical expression (Peebles 1980) for $\omega$ in case of a power-law ${ }^{2}$ like

$\xi(r)=\left(\frac{r}{r_{0}}\right)^{-\gamma}$,

( $r_{0}$ is the clustering length) namely

$\omega(\theta)=A_{\omega}\left(\frac{\theta}{1 \mathrm{rad}}\right)^{1-\gamma}$.

The angular clustering amplitude at $\theta=1 \mathrm{rad}$ is

$A_{\omega}=\sqrt{\pi} r_{0}^{\gamma} \frac{\Gamma(\gamma / 2-1 / 2)}{\Gamma(\gamma / 2)} \int_{0}^{\infty} \mathrm{d} \bar{r} p_{1}(\bar{r}) p_{2}(\bar{r}) \bar{r}^{1-\gamma}$.

However, due to the integral, $A_{\omega}$ diverges to infinity if the width of the distributions $p_{1,2}$ approaches zero and as long as $p_{1,2}$ remain overlapping; the latter is less of an issue if we are considering auto-correlations, $p_{1}=p_{2}$. To demonstrate this point, let us assume that $p_{1,2}$ are top-hat functions with centre $r_{\mathrm{c}}$ and width $2 \Delta r$ :

$p_{1,2}(r)=\frac{1}{2 \Delta r} \times \begin{cases}1 & \text { for } r \in\left[r_{\mathrm{c}}-\Delta r, r_{\mathrm{c}}+\Delta r\right] \\ 0 & \text { otherwise. }\end{cases}$

It follows for $A_{\omega}$ :

$A_{\omega} \propto \frac{1}{4 \Delta r^{2}} \int_{r_{\mathrm{c}}-\Delta r}^{r_{\mathrm{c}}+\Delta r} \mathrm{~d} \bar{r} \bar{r}^{1-\gamma} \approx \frac{1}{2 \Delta r} r_{\mathrm{c}}^{1-\gamma}$.

The last step is valid for small $\Delta r \ll r_{\mathrm{c}}$, which shows that $A_{\omega}$ diverges for narrow distributions as $\Delta r^{-1}$. This small calculation implies that Limber's equation possibly over-estimates the angular correlation $\omega$ to some degree.

In the astronomical literature, Eq. (4) is also known in other forms involving the three-dimensional power spectrum (e.g. Kaiser 1998; Hamana et al. 2004). No matter the form of Limber's equation, it always suffers from the divergence previously discussed and from the inaccuracies that are going to be discussed in the following.

\subsection{Thin-layer approximation}

Approximation (4) fails if the weight functions become deltalike functions, i.e. for galaxies being located inside one layer at comoving distance $r_{\mathrm{c}}$. To obtain the correct solution for

$p(r)=\delta_{\mathrm{D}}\left(r-r_{\mathrm{c}}\right)$

1 These approximations are accurate to about $10 \%$ for angles smaller than $\theta \lesssim 40^{\circ}$ which covers the typical range of investigated separations.

2 It has been found that a power-law $\xi$ is a fairly good description of the true spatial correlation function (e.g. Zehavi et al. 2002) of galaxies for scales below $\sim 15 \mathrm{Mpc} / \mathrm{h}$, which makes Limber's equation quite practical. Typical values are $\gamma \sim 1.8$ and $r_{0} \sim 5 h^{-1} \mathrm{Mpc}$. (where $\delta_{\mathrm{D}}(r)$ is the Dirac delta function) we simply have to go back to Eq. (1) and find for auto-correlations, $p_{1}=p_{2}$ :

$\omega(\theta)=\xi\left(r_{\mathrm{c}} \sqrt{2} \sqrt{1-\cos \theta}, r_{\mathrm{c}}\right) \approx \xi\left(r_{\mathrm{c}} \theta, r_{\mathrm{c}}\right)$.

This means that for very narrow filter we essentially observe a rescaled $\xi$. An immediate consequence is that $\omega$ has the same slope, $\gamma$, as $\xi$, if $\xi$ happens to be a power-law as in Eq. (8); on the other hand for wide filter, in the regime where Limber's approximation is accurate, $\omega$ has a shallower slope of $\gamma-1$.

It can be shown that Eq. (14) is an approximate solution of Eq. (1) for separations beyond a certain limit depending on the shape of the filter $p(r)$. This is because

$R=\sqrt{2 \bar{r}^{2}(1-\cos \theta)+\frac{\Delta r^{2}}{2}(1+\cos \theta)}$

is essentially only a function of $\bar{r}$, the left summand under the root dominates, for $\theta$ larger than

$2 \tan \left(\frac{\theta}{2}\right) \approx \theta \gg \sqrt{2} \frac{\sigma}{r_{\mathrm{m}}}$,

where the approximation of $\tan$ is accurate to $10 \%$ for $\theta \lesssim 60^{\circ}$, $\sigma$ is the $1 \sigma$-width and $r_{\mathrm{m}}$ the mean of the weight $p(r)^{3}$. In this regime, Eq. (1) simplifies to

$\omega(\theta)=\int_{0}^{+\infty} \mathrm{d} \bar{r} F(\bar{r}) \xi(\bar{r} \sqrt{2} \sqrt{1-\cos \theta}, \bar{r})$,

which is a family of thin-layer solutions (14) weighted averaged with the kernel

$F(\bar{r}) \equiv \int_{-2 \bar{r}}^{+2 \bar{r}} \mathrm{~d} \Delta r p\left(\bar{r}+\frac{\Delta r}{2}\right) p\left(\bar{r}-\frac{\Delta r}{2}\right)$.

Already for moderately wide $p(r), F(r)$ is relatively peaked, so that for large enough $\theta$, Eq. (17) is close to the solution (14) with $r_{\mathrm{c}} \approx r_{\mathrm{m}}$. Note that $F(r)$ is normalised to one.

\subsection{Numerical integration of the exact equation}

With the availability of computers there is no longer a need to use any approximations in this context. However, one finds that solving Eq. (1) by numerical integration with a power-law $\xi$ is cumbersome because many fine bins are needed to achieve the desired accuracy. It is advisable to consider a numerical integration with different coordinates such that the spatial part of $\xi$ depends just on one integration variable $R$.

This can be done by changing the integration variable in the inner integral of (1) from $\Delta r$ to $R$ :

$\omega(\theta)=\frac{1}{1+\cos \theta} \int_{0}^{\infty} \mathrm{d} \bar{r} \int_{\bar{r} \sqrt{2(1-\cos \theta)}}^{2 \bar{r}} \mathrm{~d} R Q(\bar{r}, \Delta) \xi(R, \bar{r}) \frac{R}{\Delta}$,

where

$\Delta \equiv \frac{1}{\sqrt{2}} \sqrt{\frac{R^{2}-2 \bar{r}^{2}(1-\cos \theta)}{1+\cos \theta}}$,

and

$Q(\bar{r}, \Delta) \equiv p_{1}(\bar{r}-\Delta) p_{2}(\bar{r}+\Delta)+p_{1}(\bar{r}+\Delta) p_{2}(\bar{r}-\Delta)$.

${ }^{3}$ Pairs of galaxies from this distribution will typically have $\bar{r}=\left\langle\left(r_{1}+\right.\right.$ $\left.\left.r_{2}\right) / 2\right\rangle \approx r_{\mathrm{m}}$ and for their mutual distance $\Delta r \approx \sqrt{\left\langle\left(r_{1}-r_{2}\right)^{2}\right\rangle} \approx \sqrt{2} \sigma$. 


\section{Accuracy of Limber's equation}

The aim of this section is to assess the accuracy of Limber's equation by comparing its predictions to the exact solution for the angular correlation function. For this purpose, different spatial clustering models $\xi$ were used that are constant in time (or $\bar{r}$ ). Moreover, the focus is on auto-correlations $\omega$, hence $p_{1}(r)=p_{2}(r) \equiv p(r)$.

In order to quantify the accuracy of Limber's approximation, the exact solution Eq. (1) was solved by numerical integration, by means of (19), and compared to the result from Limber's Eq. (4). In order to make a fair comparison, the same small angle approximation as in (4) was applied to the exact equation, which, as it is written in (19), does not require small galaxy separations.

\subsection{Power-law spatial correlation functions}

For spatial correlation functions such as (8), the angular clustering is for small separations a power-law with slope $\gamma-1$ and for large separations with slope $\gamma$. This was discussed in Sect. 2.3. The transition region can be estimated by the angle $\theta_{\text {break }}$ at which both approximations intersect. For a rough estimate of the intersection angle it was assumed that $p(r)$ is essentially a top-hat as in $(11)^{4}$ :

$\theta_{\text {break }} \approx \frac{2 \sqrt{3}}{\sqrt{\pi}} \frac{\Gamma(\gamma / 2)}{\Gamma(\gamma / 2-1 / 2)} \frac{\sigma}{r_{\mathrm{m}}}$.

As observed before, the point of break down of Limber's equation is a function of $\sigma / r_{\mathrm{m}}$, but also a function of the slope $\gamma$. Limber's approximation works better for larger $\gamma$ than for smaller $\gamma$.

By looking at a wide range of $\sigma / r_{\mathrm{m}}$ and realistic $\gamma$, it was found that the relative error of Limber's approximation at $\theta_{\text {break }}$ is already large, roughly $100 \%$. Therefore, the validity regime of this approximation stops well below the break position. As a rule-of-thumb, it was found that the relative error is about $10 \%$ at $\theta_{\text {break }} / 10$.

\subsection{More realistic galaxy clustering}

Although (spatial) galaxy clustering is empirically well described by a power-law on scales below $r \sim 15 \mathrm{Mpc} / \mathrm{h}, \xi(r)$ on large cosmological scales eventually adopts the shape of the dark matter clustering which does not have a constant slope $\gamma$ (e.g. Weinberg et al. 2004). Therefore, the previous discussion is only applicable if the effective physical scales, that correspond to the angular separation interval under investigation, are below $r \sim 15 \mathrm{Mpc} / \mathrm{h}$. In the opposite case, we expect that the accuracy of Limber's equation is not only a function of $\sigma / r_{\mathrm{m}}$ and an average of $\gamma$, but also a function of $r_{\mathrm{m}}$ alone.

To obtain more realistic $\xi(r)$, two representative models for galaxy clustering, based on a halo-model as in Magliocchetti $\&$ Porciani (NFW parameters; 2003), were used in this section: one $\xi$ is supposed to represent the clustering of galaxies that preferentially inhabit dark matter haloes of larger mass ( $M_{\text {halo }} \geq 10^{12.6} M_{\text {sun }}$; "red galaxies"), another $\xi$ describes galaxies that also live in smaller mass haloes $\left(M_{\text {halo }} \geq 10^{11} M_{\text {sun }}\right.$; "blue galaxies"). These two $\xi$ 's were placed at different redshifts between $0 \leq z \leq 5$, "observed" on the sky through filters $p(r)$ of

${ }^{4}$ Note that the variance of the top-hat is $\sigma=\Delta r / \sqrt{3}$, where $\Delta r$ is the width of the top-hat.

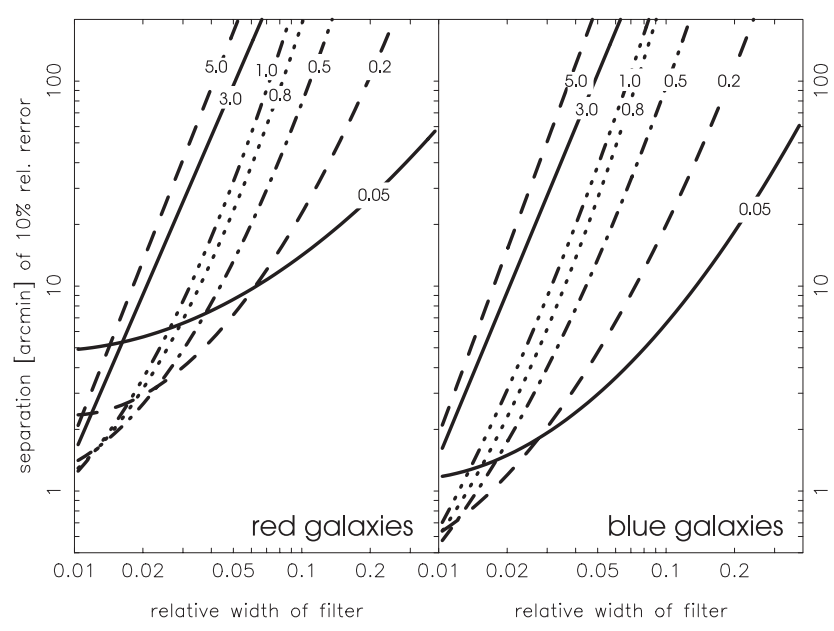

Fig. 1. Galaxy separation, as a function of $\sigma / r_{\mathrm{m}}$ of the filter, at which Limber's equation becomes inaccurate by $10 \%$ for red (left panel) and blue (right panel) galaxies. Different lines correspond to different $r_{\mathrm{m}}$; the corresponding redshifts are the line labels. Note that for estimating the relative error the local correlation function of red and blue galaxies was simply moved to higher redshifts. The (flat) fiducial cosmology assumes $\Omega_{\mathrm{m}}=0.3$.

varying widths $\sigma / r_{\mathrm{m}}$, and compared to Limber's equation in order to work out the relative error. Figure 1 shows the estimated separation limits.

The dependence of the accuracy on the centre, $r_{\mathrm{m}}$, can clearly be seen, however becoming weaker for larger $r_{\mathrm{m}}$. The difference between red and blue galaxies is most pronounced for small $r_{\mathrm{m}}$ but there are clear differences for $\sigma / r_{\mathrm{m}} \lesssim 0.02$, even for large $r_{\mathrm{m}}$. Limber's prediction of the angular correlations of red galaxies is more accurate than the predictions for blue galaxies because on megaparsec-scales, $\xi$ of red galaxies is steeper than $\xi$ of blue galaxies.

\section{Summary and conclusions}

The Limber equation is an approximate solution for the angular correlation function of objects, mainly applied in the context of galaxies, for a given spatial correlation function.

In this paper, it was shown that the assumptions made for Limber's approximation become inaccurate beyond a certain angular separation, which essentially depends on $\sigma / r_{\mathrm{m}}$ and the shape of the spatial correlation function. It should be emphasised that this inaccuracy has no relation to the flat-sky approximation (small separations) that is commonly adopted when working with Limber's equation.

Beyond what separation $\theta$ does Limber's approximation become wrong by $10 \%$ ? For power-law $\xi$ 's this separation depends entirely on $\gamma$ and $\sigma / r_{\mathrm{m}}$ of the filter (selection function) used. Spatial correlations with larger $\gamma$ are treated more accurately by Limber's equation than $\xi$ with shallower $\gamma$. As a rule-of-thumb, a systematic error of $10 \%$ is reached at about $260 \frac{\sigma}{r_{\mathrm{m}}}$ arcmin for $\gamma \sim 1.6$.

For more realistic $\xi$ 's with a running slope $\gamma$, the separation is also dependent on $r_{\mathrm{m}}$, the centre of the filter. Even for relatively wide filters with $\sigma / r_{\mathrm{m}} \sim 0.2$, Limber's equation becomes inaccurate on the $10 \%$ level at a scale of a few degrees separation. On the other hand, for narrow filters of about $\sigma / r_{\mathrm{m}} \lessgtr 0.02$, a systematic error of $10 \%$ is already reached after $\sim 10^{\prime}$ (or even a few arcmin depending on $r_{\mathrm{m}}$ ). 
Note that a filter can appear quite wide, i.e. $\sigma_{\mathrm{z}} / \bar{z}$ is large, as a function of redshift $z$ while it is actually quite narrow as a function of comoving distance, the latter being the relevant parameter for the accuracy of Limber's equation. In particular, this means that applying Limber's equation to high redshift galaxies may be a problem.

In cases where Limber's approximation is in doubt, it is suggested that one uses the exact Eq. (19) that can easily be integrated numerically.

Limber's approximation is also used in gravitational lensing to quantify correlations between the gravity-induced distortions of faint galaxy images (see e.g. Bartelmann \& Schneider 2001; Kaiser 1998). In this case, the filter function relevant for lensing is the lensing efficiency that, quite naturally, has a wide distribution; one can roughly estimate $\sigma / r_{\mathrm{m}} \approx 0.22$ if the source galaxies have a typical value of $z_{\mathrm{s}}=1$. The centre of this filter is at about $\bar{z} \approx 0.5$. These values will depend on the distribution of source galaxies in redshift and the fiducial cosmological model. Taking these values as a rough estimate of the relative lensing filter width and centre, and assuming that the dark matter clustering is somewhere between the clustering of red and blue galaxies, one can infer from Fig. 1 that a two-point auto-correlation function of the convergence, based on Limber's equation, is accurate to about $10 \%$ for separations less than several degrees; beyond that, an alternative description should be used.

Acknowledgements. I would like to thank Hendrik Hildebrandt and, in particular, Peter Schneider for carefully reading the manuscript, and their comments. This work was supported by the Deutsche Forschungsgemeinschaft (DFG) under the project SCHN 342/6-1. Furthermore, I am grateful for the kind hospitality of the Karolinska Institute, Stockholm, and David Kitz Krämer.

\section{References}

Bartelmann, M., \& Schneider, P. 2001, Phys. Rep., 340, 291

Hamana, T., Ouchi, M., Shimasaku, K., Kayo, I., \& Suto, Y. 2004, MNRAS, 347, 813

Kaiser, N. 1998, ApJ, 498, 26

Limber, D. N. 1953, ApJ, 117, 134

Magliocchetti, M., \& Porciani, C. 2003, MNRAS, 346, 186

Peebles, P. J. E. 1980, The large-scale structure of the universe, Research supported by the National Science Foundation (Princeton, N.J.: Princeton University Press), 435

Weinberg, D. H., Davé, R., Katz, N., \& Hernquist, L. 2004, ApJ, 601, 1

Zehavi, I., Blanton, M. R., Frieman, J. A., et al. 2002, ApJ, 571, 172 